\title{
BIOPHOTONICS
}

\section{Global position by polarization}

Could marine animals be using polarization-sensitive vision as a natural global positioning system that functions underwater? It's certainly feasible, according to a recent study performed by a collaboration of computer scientists, marine biologists and physicists from the US and Australia (Sci. Adv. 4, eaao6841; 2018). The team's experimental data and analysis show that the Sun's position (heading and elevation angles) can be determined from underwater polarization patterns of light, thus allowing the observer to deduce their global position if they have knowledge of the time and date. Such underwater polarization patterns are generated by a combination of the refraction and scattering of sunlight. In particular, sunlight passing through the air/water interface of the sky/sea boundary becomes preferentially polarized in the plane of the incident and refracted rays. Meanwhile, scattering events partially polarize the light perpendicular to this plane. The combination of effects creates rich polarization patterns that vary with the Sun's position.

To test their hypothesis, the team set up an underwater polarization-sensitive camera where polarization filters made from parallel aluminium nanowires aligned at angles of $0^{\circ}, 45^{\circ}, 90^{\circ}$ and $135^{\circ}$ were deposited onto the pixels of a 2-megapixel charge-coupled device image sensor. The arrangement allows the camera to capture light intensity, partial polarization and polarization angle images, and mimics the vision

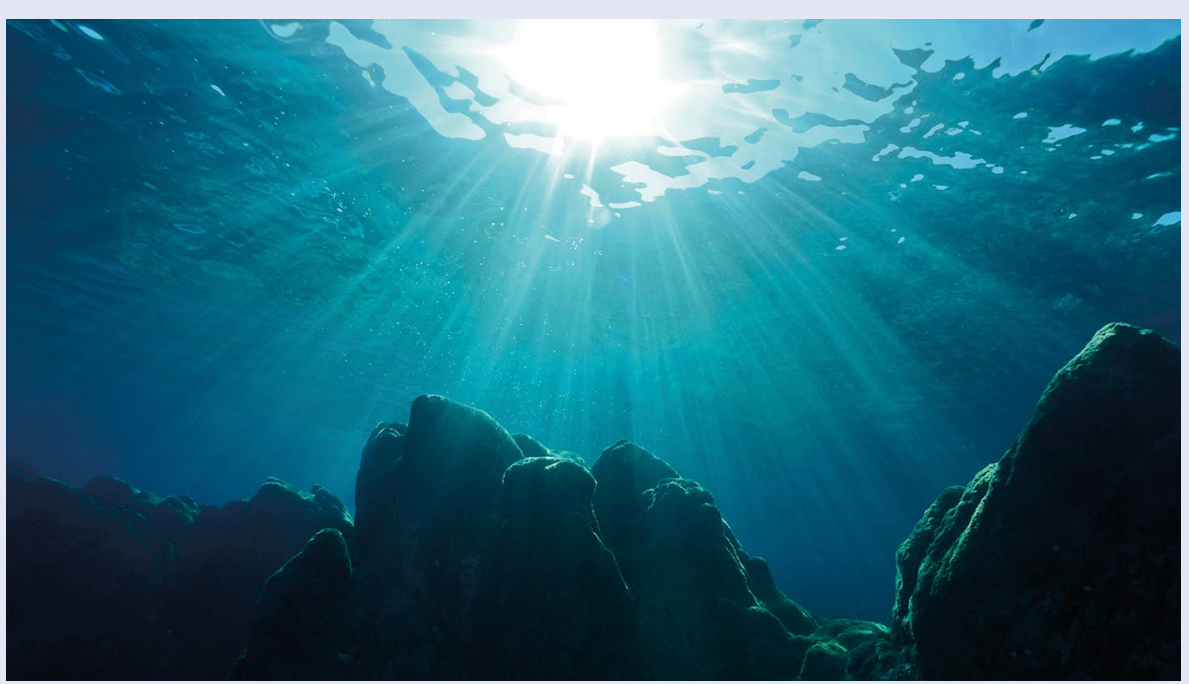

Credit: Frederic Pacorel / Alamy Stock Photo

system of the mantis shrimp. Experimental data were then captured using the camera at different locations (Australia, Hawaii, Florida and Finland) around the world and at different water depths (2-20 m) and at different times of day between sunrise and sunset. The results showed that navigation using such a system is possible with an error of $\sim 6 \mathrm{~m}$ for every $1 \mathrm{~km}$ travelled.

As several marine animals are known to have polarization-sensitive vision, it throws open the possibility that they could be using their vision as a solar compass for long-distance underwater navigation and geolocalization purposes. It is already established that many landdwelling arthropods such as ants, bees, beetles and spiders use light polarization as a navigational aid. Despite the team's measurements only having taken place at four different global locations, they believe that the uniformity of the properties of oceanic water mean that the method has general applicability.

\section{Oliver Graydon}

Published online: 29 May 2018

https://doi.org/10.1038/s41566-018-0187-3 